\title{
An intractable case of Pseudomonas aeruginosa infection after scleral buckling for rhegmatogenous retinal detachment
}

\author{
Nami Nishikiori \\ Hiroshi Ohguro \\ Department of Ophthalmology, \\ Sapporo Medical University School of \\ Medicine
}

\begin{abstract}
Background: Scleral buckling is still a common procedure to repair rhematogenous retinal detachment, and acute or chronic infection of the scleral explant is rare. We report an intractable case of acute scleral explant infection by Pseudomonas aeruginosa.

Case: A 36-year-old man suffered from acute scleral explant infection by $P$. aeruginosa fortyeight hours after scleral buckling for rhegmatogenous retinal detachment. The infection was treated by intravenous administration of various appropriate antibiotics for eighteen days and washing the scleral explant with appropriate antibiotics, and appeared to be resolved. However, three months after the initial surgery, we had to remove the scleral explant because of recurrent infection.
\end{abstract}

Observations: We encountered an intractable case of acute scleral explant infection by $P$. aeruginosa, that recurred and forced the removal of the scleral explant.

Conclusions: We found that recurrence of infection necessitated removal of the scleral explant, even though the organism was sensitive to the antibiotics used to treat the infection, and there was an appropriate duration of treatment. Early diagnosis and countermeasures, first considering conservative management, which may have a role in delaying buckle removal, and thus reduce the risk of retinal redetachment, and help prolong the time until surgical treatment such as removing the scleral explant is required.

Keywords: acute scleral explant infection, Pseudomonas aeruginosa, rhegmatogenous retinal detachment

Scleral buckling is still a common procedure to repair rhematogenous retinal detachment, and acute or chronic infection of scleral explants is rare. However, once scleral explant infection occurs, almost all cases are intractable and require the removal of the explant (Schwartz et al 1981; Roldan-Pallares et al 1999). In addition, multiple drugresistant bacteria such as methicillin-resistant Staphylococcus aureus and Pseudomonas aeruginosa have become a clinical problem (Holland et al 1991; Chaudhry NA et al 1998; Asaria et al 1999).

This is a report about a patient with intractable scleral explant infection by $P$. aeruginosa that was still sensitive to multiple antibiotics. First, appropriate antibiotics appeared to resolve the acute scleral explant infection, but three months after the initial surgery we had to remove the explant because of recurrent infection.

\section{Case report}

A 36-year-old man presented with rhematogenous retinal detachment in both eyes. On the initial examination, his best-corrected visual acuity findings were 20/20 (OD) and 20/50 (OS). Two days after the diagnosis of rhematogenous retinal detachment, 
he received uneventful scleral buckling surgery with an encircling silicone sponge band (\#506G) with a sleeve (\#70) and cryopexy for both eyes under general anesthesia. The total operation time was 110 minutes. Forty-eight hours after the surgery, he noticed chemosis of the bulbar conjunctiva and severe hyperemia in his right eye (Figure 1A). Slit-lamp examination revealed a clear looking anterior chamber, cornea, and lens. Fundus examination showed a clear view and the attached retina supported by the buckling procedure. $P$. aeruginosa, sensitive to penicillin, cephems, and carbapenem, was cultured from discharge from his right eye (Table 1). No problem was observed in his left eye. Beginning 48 hours after the surgery, he received a course of systemic antibiotics consisting of $2 \mathrm{~g} /$ day imipenem cilastatin intravenously for 4 days, $2 \mathrm{~g}$ /day cefozopran $\mathrm{HCl}$ intravenously for 7 days, and $2 \mathrm{~g}$ /day cefcapene pivoxil hydrochloride intravenously for 7 days. Simultaneously, cefmenoxime hydrochloride, levofloxacin, and sulbenicillin sodium drops were administered into the right eye six times a day for 14 days. Despite the treatments, the conjunctival chemosis and hyperemia only slightly improved, and additional purulent discharge appeared. Therefore we washed the silicone sponge explant by irrigating the buckle with exposure to $1 \mathrm{~g}$ cefazolin sodium and restoration of the conjunctiva at 20 days after the initial surgery. Then he received antibiotics consisting of $1 \mathrm{~g}$ /day meropenem trihydrate intravenously for an additional 7 days. Twenty-seven days after the initial surgery, his symptoms finally disappeared.

However, three months after the initial surgery, he experienced conjunctival chemosis, recurrent hyperemia, and the exposure of the silicone sponge explants (Figure 1B). Therefore we removed the scleral explant. Pseudomonas aeruginosa, which was sensitive to the same antibiotics as before was cultured from the removed scleral explant (Table 1). He received antibiotics consisting of $2 \mathrm{~g} / \mathrm{day}$ cefozopran $\mathrm{HCl}$ intravenously for 4 days and $400 \mathrm{mg}$ /day oral levoflozacin postoperatively. His symptoms immediately disappeared within 2 days after removal of the explant. Sixteen months after the removal the explant, the retina remained attached and the best-corrected visual acuity findings were 20/20 (OD) and 20/20 for (OS). Upon inquiry, he did not have any history of trauma or gross contamination of the ocular surface, systemic infection, or diabetes.

\section{Discussion}

The reported scleral explant infection incidence varies between $0.5 \%$ and $18 \%$, and is affected by the surgical technique, different synthetic materials of scleral explants, duration of surgery, size, and position of the buckle (Russo and Ruiz 1971; Schwartz et al 1981; Roldan-Pallares et al 1999). Patients who are operated on with scleral buckling for rhematogenous retinal detachment sometimes require removal of the implant because of infection, most commonly seen with the use of a silicone sponge (9\%) or hydrogel implant $(1.3 \%)$ and least commonly encountered with a silicon rubber band $(0.6 \%)$ (Roldan-Pallares et al 1999). Of the identified bacteria in scleral buckling infection, coagulase-negative staphylococcus is the most common, and in acute-onset scleral explant infection, which is comparatively usual, S. aureus, Proteus mirabilis, and $P$. aeruginosa are commonly identified (Russo a

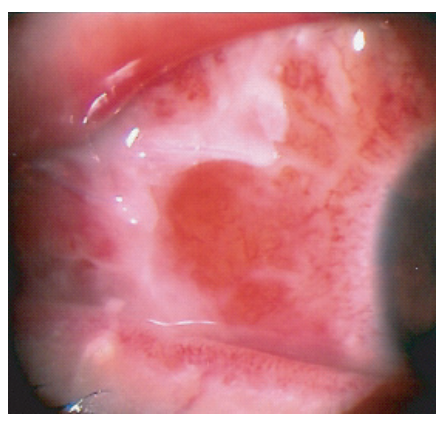

b

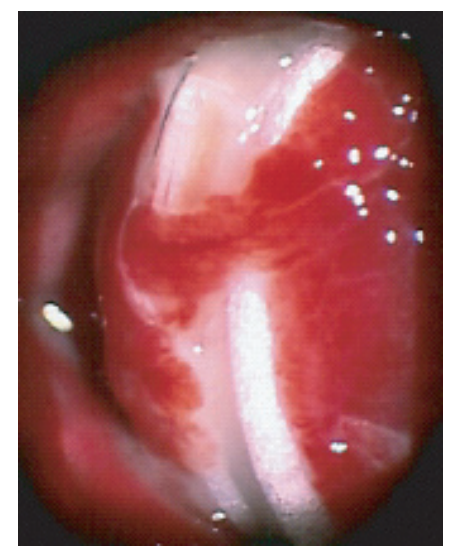

Figure IA Although the conjunctival chemosis and hyperemia improved slightly, purulent discharge appeared from the temporal position of the buckle. B Three months after the initial surgery, conjunctival chemosis and hyperemia recurred, along with exposure of the silicone sponge explants. 
Table I The sensitivity of antibiotics

\begin{tabular}{llll}
\hline Name of antibiotic & Sensitivity & Name of antibiotic & Sensitivity \\
\hline Piperacillin (PIPC) & $\mathrm{S} \delta 8$ & Sulfamethoxazole-trimethoprim (ST) & $\mathrm{N}>2$ \\
Ceftazidime (CAZ) & $\mathrm{S} \delta 2$ & Ciprofloxacin (CPFX) & $\mathrm{R} \delta 0.25$ \\
Cefoperazone: (CPZ) & $\mathrm{S} \delta 8$ & Fosfomycin (FOM) & $\mathrm{S}>\mathrm{I}$ (6 \\
Cefsulodin (CFS) & $\mathrm{S} \delta 4$ & Imipenem (IPM) & $\mathrm{S} \delta$ I \\
Aztreonam (AZT) & $\mathrm{S}=4$ & Levofloxacin (LVFX) & $\mathrm{S} \delta 0.5$ \\
Gentamicin (GM) & $\mathrm{S}=4$ & Sulbactam/cefoperazone (CPZ/SBT) & $\mathrm{S} \delta$ I6 \\
Tobramycin (TOB) & $\mathrm{S} \delta \mathrm{I}$ & Cefozopran (CZOP) & $\mathrm{S} \delta 2$ \\
Amikacin (AMK) & $\mathrm{S} \delta 4$ & Cefepime (CFPM) & $\mathrm{S} \delta 4$ \\
Minocycline (MINO) & $\mathrm{I}=8$ & Meropenem (MEPM) & $\mathrm{S} \delta \mathrm{I}$ \\
\hline
\end{tabular}

Abbreviations: S, Sensitive; I, Indistinct; R, Resistant.

and Ruiz 1971; Schwartz et al 1981; Lindsey et al 1983; Holland et al 1991; Smiddy et al 1993). The interval from the scleral explant procedure to the infection ranges from 1 to 240 months (median, 8 months and within 6 months 47\%) (Schwartz et al 1981; Roldan-Pallares et al 1999).

In this study, we found that although clinically there was an initial response to conservative management of the bucklerelated infection, it recurred, and necessitated removal of the buckle. This occurred even though the organism was sensitive to the antibiotics used to treat the infection, and there was an appropriate duration of treatment. There have been no reports that conservative management successfully treated scleral explant infection. In addition, multiple-drug-resistant $P$. aeruginosa has become a clinical problem (Holland et al 1991; Chaudhry 1998; Asaria et al 1999). In our case, the $P$. aeruginosa was sensitive to multiple antibiotics and appropriate antibiotic treatment appeared to resolve the acute scleral explant infection (Table 1), but we were finally forced to remove the explant because the infection recurred. However conservative management may have a role to play in such infections by delaying buckle removal and thus possibly reducing the risk of retinal redetachment after buckle removal (Schwartz and Pruett 1977). While some reports have suggested that removing the scleral explant causes retinal detachment (Russo and Ruiz 1971; Ulrich et al 1974; Schwartz and Pruett 1977; Schwartz et al 1981), in our case this did not occur, perhaps because we could maintain the screral explant for three months by treatment with antibiotics. To prevent scleral explant infection, one report investigated the value of soaking the scleral explant in antibiotics before placement, which resulted in a sevenfold decrease in infection (Arribas et al 1984).

If we suspect the scleral explant infection, early diagnosis and countermeasures, first considering conservative management, and the appropriate timing for surgical treatment such as exchanging the scleral explant if possible and removing the scleral explant if it is appropriate, are required. We hope that our experience will help to improve the treatment of intractable cases of acute scleral explant infection.

\section{References}

Arribas NP, Olk RJ, Schertzer M, et al. 1984. Preoperative antibiotic soaking of silicone sponges. Does it make a difference? Ophthalmology, 91:1684-9.

Asaria RH, Downie JA, McLauglin-Borlace L, et al. 1999. Biofilm on scleral explants with and without clinical infection. Retina, 19:447-50.

Chaudhry NA, Tabandeh H, Rosenfeld PJ, et al. 1998. Scleral buckle infection with ciprofloxacin-resistant Pseudomonas aeruginosa. Arch Ophthalmol, 116:1251.

Holland SP, Pulido JS, Miller D, et al. 1991. Biofilm and scleral buckleassociated infections. A mechanism for persistence. Ophthalmology, 98:933-8.

Lindsey PS, Pierce LH, Welch RB. 1983. Removal of scleral buckling elements. Causes and complications. Arch Ophthalmol, 101:570-3.

Roldan-Pallares M, del Castillo Sanz JL, Awad-El Susi S, et al. 1999. Long-term complications of silicone and hydrogel explants in retinal reattachment surgery. Arch Ophthalmol, 117:197-201.

Russo CE, Ruiz RS. 1971. Silicone sponge rejection. Early and late complications in retinal detachment surgery. Arch Ophthalmol, 85:647-50.

Schwartz PL, Maris PJ, Maris CE. 1981. Silastic sponge implants in retinal detachment surgery. Ann Ophthalmol, 13:1089-92.

Schwartz PL, Pruett RC. 1977. Factors influencing retinal redetachment after removal of buckling elements. Arch Ophthalmol, 95:804-7

Smiddy WE, Miller D, Flynn HW Jr. 1993. Scleral buckle removal following retinal reattachment surgery: clinical and microbiologic aspects. Ophthalmic Surg, 24:440-45.

Ulrich RA, Burton TC. 1974. Infections following scleral buckling procedures. Arch Ophthalmol, 92:213-15. 
